2) Large and middle sized type (8 cases): Usually, in addition to distinct localization, feeding arteries and draining veins of the malformations, main cerebral vessels were clearly demonstrated. They have tendency to develop not so severe subarachnoid hemorrhage. Total extirpation would be a treatment of choice.

3) Small arteriovenous malformations ( 8 cases): They tended to cause intracerebral hematomas, evacuation of which usually gave excellent results.

We have another subgroup (3 cases) in which angiograms revealed abnormal veins alone, though the size of the malformations corresponded to this group.

Among the above-mentioned material there were two cases which showed aneurysmal sac formation in the arteriovenous malformations.

\title{
72. Clinical Study of Cerebral Pan-Arteriography under Transient Cardiac Arrest
}

\author{
Toshio Beppu, Hidemaro Kuramitsu, Haruto Gomi, \\ Yasuharu ARai, Hiroo Hiratsuka \\ and Tomio AKrmoto \\ Surgical Department, Tokyo Women's Medical College
}

Cerebral pan-arteriography is the most important procedure in order to know the entire intra-cranial vascular system, especially in cerebral aneurysm, A-V malformation and occlusive vascular disease.

The authors have injected the contrast medium to one side of the carotid artery quickly under transient cardiac arrest and have devised a new method of obtaining a clear arteriography of the intra-cranial arterial system.

After the basic experiments of 8 mongorel dogs, we have come to the conclusion that this method could be used clinically in safety, and arteriograms of 24 cases in ages ranging from 4 years to 73 years were demonstrated with good results. Of these cases, most of the cases are traumatic cases of the head, but a severe case of Tetralogy of Fallot complicated with focal epilepsy and another Tetralogy of Fallot together with cerebral abscess were observed with no complication.

The patient lies in supine position with the apparatus for ECG and the indwelling intravenous catheter is inserted in the cubital vein and Thiopental is administered for general anesthesia. Immediately afterwards, the needle 
or the indwelling intra-arterial catheter inserted in the right carotid artery percutaneously. Then, while ECG is being monitored continuously, 6-8 mg per $\mathrm{kg}$. weight of acethylcholine is injected with full force into the cubital vein and 6-13 min. later, transient ventricular asystole appear for 3-9 seconds is observed. During the circulatory asystole, the contrast medium is in jected quickly into the right carotid artery which has been prepared before. Xray of the head is taken after the injection and immediately afterwards, for the sake of safety, oxygen inhalation is done for hyperventilation to return to nomal condition spontaneously. Sinus rhythm is resumed rather early. ST changes are recovered in about 8 minutes. Atropin was not needed.

Angiography under circulatory arrest was done 41 times. Of these, the contrast medium passed through the Willis ring 27 times, besides the left side of carotid arterial system, the basilarvertebral arterial system was also observed. In adults, $30 \mathrm{cc}$ of contrast medium was sufficient. The rest of the not well visualized cases or cases that were not visualized at all were due perhaps to the bad timing of the circulatory arrest and injection of contrast medium, or the anomalie of Willis ring.

Side effects such as hypersecretion of the oral and nasal cavities were decreased by atropin intramuscular injection 30 minutes before the procedure, cough attacks due to bronchospasms caused by acetylcholine which may occassionally occur, may be stopped by deep anesthesia or by S.C.C. intravenously. These were no severe complication but transient hemiparesis for 1 hour was noticed in one case and subcutaneous hematoma caused by injection was found in 5 cases only.

\title{
73. Designation of the Arteries shown in the Vertebral Angiography
}

\author{
Kazuo Hara, Yutaka Kuru, Yasusada Fujino, \\ Hayao Shiga and Tetsuo Gamo \\ Department of Radiology, Medical School, Osaka University
}

For a detail description of the vertebral angiographic findings a simple designation of the arteries is required. The authors give the designation $V$, $B, S$ and $P$ respectively to the vertebral, the basilar, the superior cerebellar and the posterior cerebral artery. Corresponding to turning or branching of each artery, a small number is added to the initial: $B_{1}, S_{1}$, etc. The 